\title{
Impactos da pandemia de COVID-19 sobre o tratamento para cessação do tabagismo
}

\section{Impacts of the COVID-19 pandemic on smoking cessation treatment}

\author{
Maria Vanessa Dias da Silva* (D) \\ Murilo Branco Andrade \\ Monteiro \\ André Bezerra de Sena (iD \\ Petrônio José de Lima Martelli (iD \\ Isabela de Lucena Heráclio \\ Albanita Gomes da Costa de \\ Ceballos
}

\begin{abstract}
RESUMO
Introdução: A pandemia do novo coronavírus teve repercussões no funcionamento dos sistemas de saúde do mundo inteiro. 0 tabagista foi um grupo diretamente afetado por essas mudanças. Objetivo: Mensurar esse impacto a partir da análise dos dados do Programa Estadual de Controle ao Tabagismo de Pernambuco. Método: Estudo descritivo transversal, utilizando como unidades de análise dados da estratégia de monitoramento do Programa Estadual de Controle ao Tabagismo da Secretaria Estadual de Saúde de Pernambuco (SES-PE), comparando os atendimentos no segundo quadrimestre dos anos de 2019 e 2020. Resultados: Entre maio e agosto de 2019, 3.282 pacientes tabagistas buscaram tratamento para cessação do tabagismo no SUS, em Pernambuco. Em período similar, entre os meses de maio e agosto do ano de 2020, o tratamento para cessação do tabagismo foi procurado por 680 usuários tabagistas, representando uma queda de $79,28 \%$. Além disso, o número de municípios oferecendo tratamento para cessação do tabagismo no SUS caiu de 97 para 36 (62,89\%) e o número de unidades de saúde da atenção básica realizando tratamento para cessação do tabagismo no SUS de 277 para $80(71,11 \%)$. Conclusões: A diminuição da oferta do tratamento pelo Programa Estadual de Combate ao Tabagismo é preocupante. Ainda que a sua relação com a COVID-19 não esteja completamente elucidada, a cessação do uso do tabaco traz benefícios já bem estabelecidos. Dessa forma, é necessário incentivar a adoção de novas estratégias e tecnologias, aproveitando a janela de oportunidade que o temor da associação COVID-19/tabagismo criou.
\end{abstract}

PALAVRAS-CHAVE: Campanhas para o Controle do Tabagismo; COVID-19; Acesso aos Serviços de Saúde; Abandono do Uso de Tabaco; Atenção Básica

\begin{abstract}
Introduction: The new Coronavirus pandemic has had an impact on health systems worldwide. Smokers were directly affected by these changes. Objective: To measure the new Coronavirus pandemic impact on smoking cessation from the analysis of data from the Pernambuco State Tobacco Control Program. Method: Descriptive cross-sectional study, using data from the monitoring strategy of the State Tobacco Control Program of the State Health Secretariat of Pernambuco (SES-PE) as the unit of analysis, comparing services in the second quarter of 2019 and 2020. Results: Between May and August 2019, 3.282 smoking patients sought treatment for smoking cessation in SUS, in Pernambuco. In a similar period, between the months of May and August of the year 2020, treatment for smoking cessation was sought by 680 smoking users, representing a drop of $79,28 \%$. In addition, the number of municipalities offering treatment for smoking cessation in SUS dropped from 97 to $36(62,89 \%)$ and the number of primary health care units providing treatment for smoking cessation in SUS went from 277 to $80(71,11 \%)$. Conclusions: The decrease in the offer of treatment by the State Program to Combat Smoking is worrying. Although its relation with COVID-19 has not been fully elucidated, the cessation of tobacco use has already established benefits. Thus, it is necessary to encourage the adoption of new strategies and technologies, using the window of opportunity that the fear of COVID-19/smoking association created.
\end{abstract}

KEYWORDS: Anti-Smoking Campaign; COVID-19; Health Services Accessibility; Tobacco Use Cessation; Primary Care 


\section{INTRODUÇÃO}

O surgimento de um novo tipo de coronavírus, o SARS-CoV 2 (Severe Acute Respiratory Syndrome Coronavirus 2), identificado pela primeira vez em Wuhan, na China, em 2019, levou a uma pandemia com repercussões mundiais. Na tentativa de evitar colapsos nos sistemas de saúde, foram adotadas medidas de isolamento social para diminuir o número de casos em todos os continentes. Dentre elas, citam-se as restrições de viagens internacionais, limitações de circulação, toque de recolher e fechamento de estabelecimentos comerciais. Tal impacto se estendeu, inclusive, aos serviços de saúde, que tiveram ambulatórios e procedimentos eletivos reduzidos ${ }^{1}$.

Nesse contexto, segundo dados apresentados no relatório "Tratamento do Tabagismo no SUS durante a pandemia de COVID-19", divulgado pelo Instituto Nacional de Câncer José Alencar Gomes da Silva (INCA), houve redução expressiva no número de tabagistas em tratamento no Sistema Único de saúde (SUS) no país. Tal redução é explicada por fatores relacionados à diminuição de procura, bem como à redução na oferta aos serviços. Segundo o órgão, o afastamento de profissionais de saúde idosos e os casos de COVID-19 entre os trabalhadores do SUS, associados ao receio da população de frequentar as unidades de saúde, contribuíram para explicar os dados ${ }^{2,3}$.

O tabagista em isolamento social apresenta alguns riscos, como demonstrado em um estudo com 3.632 pessoas na Bélgica, o qual analisou o uso de álcool, tabaco e Cannabis durante o lockdown no país, sendo observado um aumento marginal no uso de tabaco. Os mais expostos foram indivíduos jovens, com baixa escolaridade e aqueles com trabalhos relacionados ao enfrentamento da pandemia ${ }^{4}$.

Faz-se necessário pontuar, entretanto, que o consumo do tabaco não só está relacionado a um aumento da mortalidade geral, devido a inúmeros problemas sociais e de saúde bem estabelecidos na literatura ${ }^{5,6}$, como também possivelmente a formas mais graves e com piores desfechos da COVID-19, pois foi comprovada a relação entre o tabagismo e a redução de mecanismos de defesa pulmonares ${ }^{7,8}$. Nesse sentido, o consumo de cigarro durante a pandemia deve ser pauta das autoridades sanitárias. A África do Sul, por exemplo, proibiu a venda de produtos não essenciais durante o período de lockdown, incluindo o cigarro, fato elogiado por alguns especialistas, que reforçaram, ainda, a necessidade de oferecer, associadamente, suporte para a cessação do tabagismo.

No Brasil, entre as 26 capitais dos estados e o Distrito Federal, a prevalência do tabagismo é de $12,3 \%$ no sexo masculino e de $7,7 \%$ no feminino. Dentre as capitais, Porto Alegre tem o maior percentual de fumantes entre a sua população $(14,6 \%)$ e Teresina, o menor $(4,4 \%)^{10}$. Apesar dessa prevalência considerável, o consumo do tabaco vem sofrendo duros golpes no país nas últimas décadas. Isso porque o enfrentamento ao tabagismo no Brasil é considerado uma referência pela Organização Mundial da Saúde $(\mathrm{OMS})^{11}$. Dentre as ações adotadas pelo país, destacam-se a criação de campanhas antifumo, medidas que restringem a publicidade de cigarros, criação de ambientes livres de fumo e tratamento gratuito oferecido pelo SUS ${ }^{12}$.
No Brasil, em 2005, a implantação da Convenção-Quadro da OMS para Controle do Tabaco (CQCT/OMS) tornou a Política Nacional de Controle do Tabaco, uma política de Estado. A partir disso, a prevalência de fumantes no país caiu de $15,7 \%$ para $9,8 \%$ entre 2006 e 2019. 0 tratamento para cessação de fumar do SUS juntamente com as ações educativas e a aprovação de leis relacionadas à prevenção do tabagismo foram fundamentais para isso ${ }^{13}$.

O Programa Nacional de Controle do Tabaco (PNCT) é coordenado pelo INCA, no entanto, as secretarias estaduais de saúde possuem coordenações próprias que oferecem suporte aos municípios. Tal fato baseia-se na busca pela descentralização e intersetorialidade. É válido ressaltar, ainda, que as ações contemplam não só medidas que visem diminuir a iniciação no fumo, bem como o tratamento do tabagismo, visando a integralidade do cuidado².

Em Pernambuco, estado cuja capital tem prevalência de 7,9\% de fumantes ${ }^{10}$, as medidas para combate do coronavírus atingiram 0 funcionamento de diversas Unidades de Saúde da Família (USF) e de Centros de Atenção Psicossocial (CAPS), ofertantes de tratamento para a cessação do tabagismo. Dessa maneira, é interessante analisar o impacto da pandemia da COVID-19 no Programa Estadual de Combate ao Tabagismo.

Este estudo objetivou analisar os impactos sobre pacientes tabagistas e sobre os serviços de referência para tratamento no estado de Pernambuco (PE) no segundo quadrimestre de 2019 e no segundo quadrimestre de 2020, período escolhido uma vez que em Pernambuco as medidas de isolamento social se iniciaram no mês de março.

\section{MÉTODO}

Estudo descritivo transversal, utilizando como unidades de análise, dados da estratégia de monitoramento do Programa Estadual de Controle ao Tabagismo da Secretaria Estadual de Saúde de Pernambuco (SES-PE), desenvolvida pela coordenação estadual, a partir de instrumento respondido pelas Secretarias Municipais de Saúde. Tal instrumento alimenta o Relatório Quadrimestral do Programa Nacional para Cessação do Tabagismo no SUS, recurso disponibilizado pelo INCA para auxiliar as Secretarias Estaduais e Municipais de Saúde, na consolidação do perfil dos atendimentos realizados nas unidades que ofertam o tratamento para cessação do tabagismo no SUS.

Foram selecionadas algumas variáveis para compor o estudo, dentre todas as presentes no Relatório Quadrimestral do PNCT/ $\mathrm{PE}$, de acordo com a estratificação dos Blocos de variáveis:

Bloco 1 - informações do município: estado; regional de saúde; município; situação (em funcionamento ou reiniciando).

Bloco 2 - informações dos pacientes: número de pacientes que buscaram tratamento; por sexo; por faixa etária (< 18 anos, 18-59 anos e $\geq 69$ anos). 
Bloco 3 - perfil das unidades: número de unidades de saúde que realizaram atendimento no período; tipo da unidade.

Bloco 4 - informações dos atendimentos realizados: número de pacientes atendidos na $1^{\mathrm{a}}$ avaliação clínica; número de pacientes que participaram da $1^{\text {a }}$ sessão; número de pacientes que participaram da $4^{\mathrm{a}}$ sessão; número de participantes de grupos de manutenção; número de pacientes que usaram algum medicamento para tratamento do tabagismo.

Para a análise percentual de decréscimo, foi realizado o seguinte cálculo: 100- [(variável referente ao ano de 2020×100)/variável referente ao ano de 2019]. Os resultados obtidos foram arredondados de acordo com a norma ABNT NBR 5891:2014 mantendo até duas casas decimais.

Essa pesquisa foi aprovada no Comitê de Ética em Pesquisa do Centro de Ciências da Saúde da Universidade Federal de Pernambuco (CEP/CCS/UFPE), sob o parecer de $n^{\circ} 4.652 .226$ e CAAE $n^{\circ}$ 45065621.1.0000.5208. Como os dados utilizados têm como fonte banco com dados agregados, não há possibilidade de identificação individual dos pacientes em acompanhamento no programa.

\section{RESULTADOS}

Entre maio e agosto de 2019, somando-se os pacientes nas três faixas etárias em estudo (< 18 anos, entre 18 e 60 anos, e > 60 anos), encontramos 3.282 pacientes que buscaram tratamento para cessação do tabagismo no SUS, em PE. Desses, 1.317 eram do sexo masculino, 1.825 eram do sexo feminino e 140 de sexo não informado. Quarenta e dois pacientes estavam na faixa etária menor de 18 anos de idade, 2.288 pacientes estavam entre 18 e 60 anos e 952 pacientes estavam na igual ou superior a 60 anos de idade (Tabela).

Em período similar, entre os meses de maio e agosto do ano de 2020, o tratamento para cessação do tabagismo foi procurado por 680 usuários tabagistas em $\mathrm{PE}$, sendo 312 pacientes do sexo masculino e 368 do sexo feminino. Quatorze pacientes estavam na faixa etária menor de 18 anos, enquanto 535 estavam entre 18 e 60 anos e 115 estavam na de 60 anos de idade ou mais, além de 16 pacientes com a idade não informada.

No intervalo considerado, em 2019, havia unidades de saúde realizando tratamento para cessação do tabagismo no SUS em 97 municípios, sendo 277 unidades na Atenção Básica, 12 unidades de CAPS e seis unidades de atendimento especializado.

Entre os meses de maio e junho de 2020, os usuários encontraram unidades realizando tratamento para cessação do tabagismo no SUS em 36 municípios, sendo 80 unidades na Atenção Básica, seis CAPS e oito unidades de atendimento especializado.

Entre maio e agosto de 2019, 3.069 pacientes realizaram a primeira avaliação clínica para cessação do tabagismo. 0 número de pacientes participando da primeira sessão e da quarta sessão de abordagem intensiva foram 2.033 e 1.357 , respectivamente. Havia 1.325 usuários tabagistas em tratamento nas sessões de manutenção e 2.086 usuários que utilizavam algum medicamento associado ao tratamento.

No mesmo intervalo, em 2020, 200 usuários conseguiram frequentar as sessões de manutenção (decréscimo de $84,90 \%$ em comparação com 2019), e 441 pacientes mantiveram o tratamento medicamentoso (decréscimo de 78,85\% em comparação com o mesmo período). Enquanto isso, 639 pacientes foram atendidos na primeira avaliação clínica para cessação do tabagismo (decréscimo de $79,17 \%$ comparado ao ano anterior). 0 número de pacientes participando da primeira sessão de abordagem intensiva foi 590 e, da quarta sessão, 463 (decréscimo de 70,97\% e 65,88\% respectivamente quando comparado ao ano de 2019) (Figura).

\section{DISCUSSÃO}

O atual surto de COVID-19 resultou na declaração de pandemia pela OMS devido a sua gravidade e propagação mundial ${ }^{14}$. A busca por soluções para mitigar os impactos sanitários e socioeconômicos da pandemia resultaram na identificação de grupos potencialmente mais vulneráveis à transmissão e às complicações de saúde pela doença. Entre esses grupos, estão os tabagistas, que, no mundo, representam cerca de 1 bilhão de pessoas e, no Brasil, mais de 20 milhões $^{15}$.

Tabela. Caracterização dos pacientes tabagistas que buscaram tratamento no SUS e da oferta do tratamento nos segundos quadrimestres de 2019 e 2020.

\begin{tabular}{|c|c|c|c|c|}
\hline & & Maio-agosto/2019 & Maio-agosto/2020 & $\begin{array}{l}\text { Decréscimo } \\
2020-2019(\%)\end{array}$ \\
\hline \multicolumn{2}{|c|}{ Tabagistas que buscaram tratamento no SUS em Pernambuco } & 3.282 & 680 & $-79,28 \%$ \\
\hline \multirow{2}{*}{ Sexo } & Masculino & 1.317 & 312 & $-76,31 \%$ \\
\hline & Feminino & 1.825 & 368 & $-79,84 \%$ \\
\hline \multirow{3}{*}{ Faixa etária } & $<18$ anos & 42 & 14 & $-66,67 \%$ \\
\hline & $18-59$ anos & 2.288 & 535 & $-76,62 \%$ \\
\hline & $\geq 60$ anos & 952 & 115 & $-87,92 \%$ \\
\hline \multicolumn{2}{|c|}{$\mathrm{N}^{\circ}$ de municípios oferecendo tratamento para cessação do tabagismo no SUS } & 97 & 36 & $-62,89 \%$ \\
\hline \multicolumn{2}{|c|}{$\begin{array}{l}N^{\circ} \text { de unidades de atenção básica realizando tratamento para cessação do tabagismo no } \\
\text { SUS }\end{array}$} & 277 & 80 & $-71,11 \%$ \\
\hline
\end{tabular}

Fonte: Elaborada pelos autores com dados dos Relatórios Quadrimestrais do Programa de Tratamento para Cessação do Tabagismo no SUS 2019 e 2020 (2021).

SUS: Sistema Único de Saúde. 


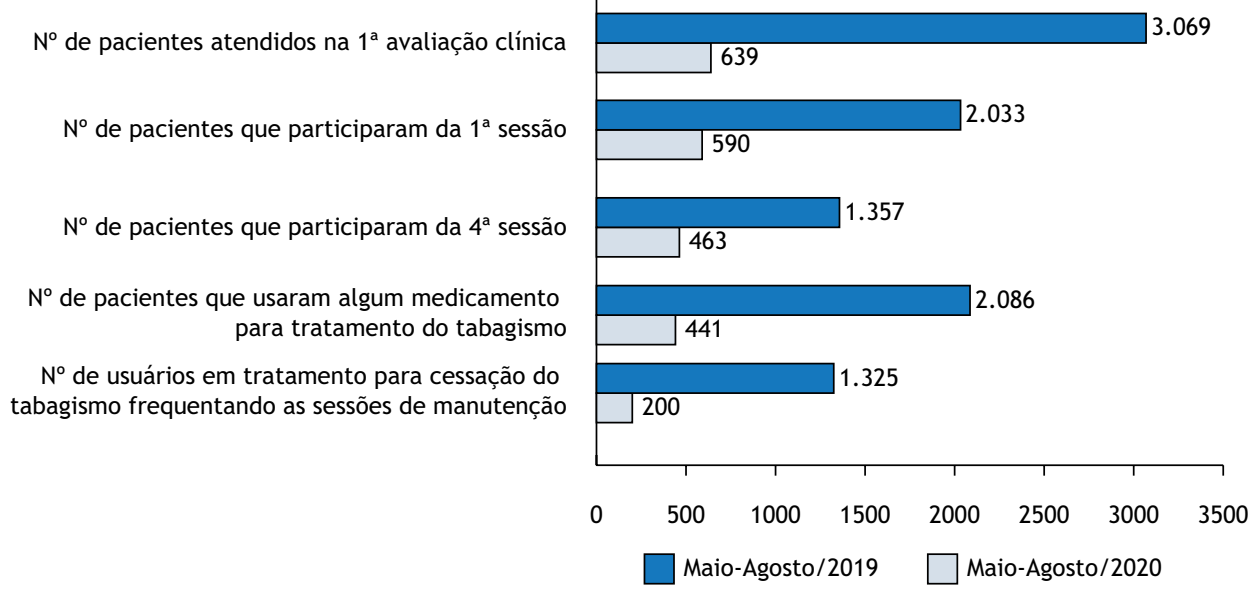

Fonte: Elaborada pelos autores com dados dos Relatórios Quadrimestrais do Programa de Tratamento para Cessação do Tabagismo no SUS 2019 e 2020 (2021).

Figura. Pacientes com o acesso ao tratamento nos segundos quadrimestres de 2019 e 2020.

A literatura ainda é conflitante quanto à relação entre a COVID19 e o tabagismo. Fumar é considerado um fator de pior prognóstico para infecções respiratórias ${ }^{16}$. Porém, a nicotina pode ter um efeito sobre os receptores da angiotensina, os quais são implicados na fisiopatologia da infecção pelo novo Coronavírus ${ }^{17}$. A ativação desses receptores estimula mudanças epigenéticas, que podem ser responsáveis pelo dano pulmonar ${ }^{18}$. Assim, 0 ato de fumar induz uma regulação positiva dose-dependente desses receptores ${ }^{19}$ e aumenta a gravidade da doença associada à resposta inflamatória ${ }^{20}$. Entretanto, aumentos na expressão dos receptores da angiotensina talvez atenuem o risco de desenvolver dano maciço ao pulmão e resposta sistêmica exagerada, as quais caracterizam formas graves da COVID-1921. Além disso, a nicotina ainda parece ter um potencial efeito anti-inflamatório ${ }^{22}$.

Apesar de tais dados conflitantes do ponto de vista fisiopatológico, a pandemia trouxe uma janela de oportunidade excelente para o combate ao tabaco. A disseminação das notícias de que tal prática eleva os níveis de mortalidade entre os doentes, em consonância com artigos mostrando um aumento em até $14 x$ comparando com a população geral ${ }^{23}$, gerou um efeito positivo na interrupção do tabagismo. Um estudo em uma clínica de cessação de tabagismo na Turquia que acompanhava 357 pacientes demonstrou uma taxa de cessação do tabaco $8 \%$ maior entre os anos de 2020 e 2021 comparado com o mesmo período nos anos anteriores, sem nenhum tratamento adicional ${ }^{24}$.

Outro estudo, feito pela Universidade de Vermont com 345 fumantes, demonstrou que a pandemia levou cerca de um quarto dos entrevistados a reduzir o uso de tabaco e em mais de um terço houve aumento na motivação para interrupção dessa prática. Além disso, mais de $20 \%$ dos entrevistados relataram cessação do tabagismo para reduzir o risco de danos causados pela COVID-1925.

Contudo, percebe-se que a pandemia da COVID-19 teve um grande impacto no Programa Estadual de Controle do Tabagismo de Pernambuco, tendo como resultado a diminuição do fornecimento de tratamento em unidades da atenção básica (queda de cerca de 71,11\% de maio-agosto/2019 para maio-agosto/2020) e, por consequência, um menor número de indivíduos contemplados. Prova disso é que o número de tabagistas procurando tratamento caiu de 3.282 , no período estudado em 2019, para 680, no intervalo similar em 2020.

Nesse cenário, em 2019, 2.086 pacientes utilizavam algum medicamento em seus tratamentos e, em 2020, o número caiu para 441. Diferentes estratégias farmacológicas estão disponíveis para tratar a dependência do tabagismo, que devem estar associadas a estratégias não farmacológicas ${ }^{26}$. Podemos presumir que o impacto da pandemia pela COVID-19 também dificultou o acesso ao medicamento pelo SUS, prejudicando o tratamento de vários usuários que dependem exclusivamente desse Sistema.

No Brasil, em um estudo transversal produzido pela Fundação Oswaldo Cruz (Fiocruz) em parceria com a Universidade Federal de Minas Gerais (UFMG) e a Universidade Estadual de Campinas (Unicamp), que avaliou hábitos de vida de mais de 45 mil pessoas durante a pandemia, observou-se que $34 \%$ dos fumantes passaram a consumir mais cigarros por dia ${ }^{27}$. Tais dados são conflitantes com as referências supracitadas, demonstrando a dependência e a importância que esses programas apresentam no combate ao cigarro e como a interrupção do acompanhamento desses pacientes pode ser prejudicial e devastadora, ratificando a necessidade de manutenção dessas políticas e criação de novas estratégias com suas devidas adequações ao período.

Essa divergência entre os dados encontrados precisa ser mais bem estudada para se conseguir compreender os motivos que levam uma população a diminuir o uso do cigarro em detrimento a um maior consumo de outras. Alguns pontos podem ser sugeridos, como: educação em saúde, acesso à informação ou funcionamento do serviço de saúde de cada região. Porém, é possível que ainda seja cedo para afirmar categoricamente a causa de tais diferenças, sendo necessárias novas avaliações retrospectivas do cenário. 
Nesta conjuntura de novos desafios ao SUS, a efetivação de ações intersetoriais na atenção primária à saúde pode aumentar o sucesso de intervenções clínicas de cessação do tabagismo ${ }^{28}$. Nesse sentido, é importante notar que o acesso ao tratamento é altamente desigual ${ }^{29}$. Observa-se que a atenção básica, em consonância com sua atribuição de porta de entrada, tem grande relevância no Programa Estadual de Controle do Tabagismo de Pernambuco. No intervalo considerado em 2019, 94\% dos atendimentos ocorreram na atenção primária; do mesmo modo, no intervalo estudado em 2020, $85 \%$ das unidades que realizaram tratamento para a cessação do tabagismo estavam na atenção primária, comprovando a desigualdade de acesso.

Existem evidências científicas robustas demonstrando que intervenções abrangentes para a cessação do tabagismo são efetivas e essenciais ${ }^{30}$. No Programa Estadual de Controle do Tabagismo de Pernambuco, oferece-se um tratamento com duração total recomendada de 12 meses que inclui as etapas de avaliação, intervenção e manutenção da abstinência. A etapa de avaliação é o momento em que é possível conhecer a história de tabagismo do paciente (incluindo idade de início e tentativas prévias de cessação), história patológica (presença ou ausência de doenças relacionadas ao fumo), avaliação da dependência de nicotina (Teste de Fagerström) e estágios de motivação para iniciar o tratamento. Essa avaliação preliminar permite ao profissional de saúde determinar se, além da abordagem cognitivo-comportamental, o paciente precisará utilizar algum tipo de medicamento ${ }^{31}$.

\section{CONCLUSÕES}

Fica evidente que a COVID-19 trouxe transtornos para a saúde pública em uma escala bem mais ampla que a prevista. A diminuição expressiva da oferta do tratamento pelo Programa Estadual de Combate ao Tabagismo nos municípios, como exposto no estudo, é reflexo do problema. Ainda que a sua relação com a COVID-19 não esteja completamente elucidada, a cessação do uso do tabaco traz benefícios já bem estabelecidos.

Dessa forma, é necessário incentivar a adoção de novas estratégias e tecnologias, visando a continuidade do cuidado com o usuário do SUS. Aproveitando, assim, a janela de oportunidade criada pelo maior temor de casos mais severos relacionados à associação COVID/tabagismo.

0 presente artigo baseia-se em dados secundários fornecidos pela Secretaria Estadual de Saúde a partir de informações oriundas das Secretarias Municipais de Saúde. Dessa forma, fatores locais específicos que prejudiquem o funcionamento da máquina pública municipal, como a própria pandemia, bem como as eleições municipais do ano de 2020, podem ter levado a subnotificações dos dados do segundo trimestre de 2020.

\section{REFERÊNCIAS}

1. Governo do Estado de Pernambuco. Decreto $N^{\circ} 48.809$, de 14 de março de 2020. Regulamenta, no estado de Pernambuco, medidas temporárias de enfretamento da emergência de saúde pública decorrente do coronavírus, conforme previsto na lei federal $N^{\circ} 13.979$, de 6 de fevereiro de 2020. Diário Oficial do Estado. 15 mar 2020.

2. Instituto Nacional do Câncer - INCA. Programa Nacional de Controle do Tabagismo. Brasília: Ministério da Saúde; 2021[acesso 11 set 2021]. Disponível em: https: / /www. inca.gov.br/imprensa/inca-aponta-reducao-de-mais-de-60no-numero-de-tabagistas-em-tratamento-no-sus-durante

3. Gandra A. Número de tabagistas em tratamento do SUS cai 66\% durante pandemia. Agência Brasil. 25 ago 2021 [acesso 11 set 2021]. Disponível em: https://agenciabrasil.ebc. com.br/saude/noticia/2021-08/numero-de-tabagistas-emtratamento-no-sus-cai-66-durante-pandemia

4. Vanderbruggen N, Matthys F, Van Laere S, Zeeuws D, Santermans L, Van den Ameele $S$ et al. Self-reported alcohol, tobacco, and Cannabis use during COVID-19 lockdown measures: results from a web-based survey. Eur Addict Res. 2020;26(6):309-15. https://doi.org/10.1159/000510822

5. Nash SH, Liao LM, Harris TB, Freedman ND. Cigarette smoking and mortality in adults aged 70 years and older: results from the NIH-AARP cohort. Am J Prev Med. 2017;52(3):276-83. https://doi.org/10.1016/j.amepre.2016.09.036

6. Pinto M, Bardach A, Palacios A, Biz A, Alcaraz A, Rodriguez B et al. Carga do tabagismo no Brasil e benefício potencial do aumento de impostos sobre os cigarros para a economia e para a redução de mortes e adoecimento. Cad Saúde Pública. 2019;35(8):1-19. https://doi.org/10.1590/0102-311X00129118

7. Patanavanich R, Glantz SA. Smoking is associated with COVID-19 progression: a meta-analysis. Nicotine Tob Res. 2020;22(9):1653-6. https://doi.org/10.1093/ntr/ntaa082

8. Vardavas $\mathrm{Cl}$, Nikitara K. COVID-19 and smoking: a systematic review of the evidence. Tob Induc Dis. 2020;18:20. https://doi.org/10.18332/tid/119324

9. Egbe CO, Ngobese SP. COVID-19 lockdown and the tobacco product ban in South Africa. Tob Induc Dis. 2020;18:1-2. https://doi.org/10.18332/tid/120938

10. Ministério da Saúde (BR). Vigitel Brasil 2019: vigilância de fatores de risco e proteção para doenças crônicas por inquérito telefônico: estimativas sobre frequência e distribuição sociodemográfica de fatores de risco e proteção para doenças crônicas nas capitais dos 26 estados brasileiros e no Distrito Federal em 2019. Brasília: Ministério da Saúde; 2020[acesso 10 mar 2021. Disponível em: http://bvsms.saude.gov.br/bvs/ publicacoes/vigitel_brasil_2019_vigilancia_fatores_risco.pdf

11. World Health Organization - WHO. WHO report on the global tobacco epidemic, 2019. Geneva: World Health Organization; 2019[acesso 10 mar 2021. Disponível em: http://www.who.int/tobacco/mpower/offer/en/

12. Ministério da Saúde (BR). O controle do tabaco no Brasil: uma trajetória. Brasília: Ministério da Saúde; 2012[acesso 10 
mar 2021. Disponível em: http://bvsms.saude.gov.br/bvs/ publicacoes/exposicao_controle_tabaco_brasil_trajetoria.pdf

13. Cavalcante TM, Mendes FL, Perez CA, Carvalho AOR, Teixeira APL, Viegas JRR. Como a Política Nacional de Controle do Tabaco pode ajudar no enfrentamento da pandemia da COVID-19? Rev Bras Cancerol. 2020;66:1-4. https://doi.org/10.32635/2176-9745.RBC.2020v66nTemaAtual.1023

14. Chatkin J, Godoy I. Tabagismo, poluição ambiental e condições climáticas são fatores de risco para COVID-19? J Bras Pneumol. 2020;46(6):1-6. https://doi.org/10.36416/1806-3756/e20200183

15. Cavalcante TM, Perez CA, Mendes FL, Machado AT, Rangel EC, Pereira RCA et al. COVID-19 e tabagismo: aspectos epidemiológicos, biológicos, psicossociais e implicações para a Política Nacional de Controle do Tabaco. Rev Bras Cancerol. 2020;66:1-10.

16. Sales MPU, Araújo AJ, Chatkin JM, Godoy I, Pereira LFF, Castellano MVC et al. Atualização na abordagem do tabagismo em pacientes com doenças respiratórias. J Bras Pneumol. 2019;45(3):1-17. https://doi.org/10.1590/1806-3713/e20180314

17. Berlin I, Thomas D, Le Faou AL, Cornuz J. COVID-19 and smoking. Nicotine Tob Res. 2020;22(9):1650-2. https://doi.org/10.1093/ntr/ntaa059

18. Cai G. Bulk and single-cell transcriptomics identify tobacco-use disparity in lung gene expression of ACE2, the receptor of 2019-nCov. MedRxiv. 2020:1-15. https://doi.org/10.1101/2020.02.05.20020107

19. Cai G, Bossé $\mathrm{Y}$, Xiao F, Kheradmand F, Amos $\mathrm{Cl}$. Tobacco smoking increases the lung gene expression of ACE2, the receptor of SARS-CoV-2. Am J Resp Crit Care Med. 2020;201(12):1557-9. https://doi.org/10.1164/rccm.202003-0693LE

20. Engin $A B$, Engin ED, Engin A. Two important controversial risk factors in SARS-CoV-2 infection: obesity and smoking. Environ Toxicol Pharmacol. 2020;78:1-8. https://doi.org/10.1016/j.etap.2020.103411

21. Lippi G, Sanchis-Gomar F, Henry BM. Active smoking and COVID-19: a double-edged sword. Eur J Intern Med. 2020;77:123-4. https://doi.org/10.1016/j.ejim.2020.04.060

22. Polosa R, Caci G. COVID-19: counter-intuitive data on smoking prevalence and therapeutic implications for nicotine. Intern Emerg Med. 2020;15(5):853-6. https: //doi.org/10.1007/s11739-020-02361-9

23. Liu W, Tao ZW, Wang L, Yuan M-L, Liu K, Zhou L et al. Analysis of factors associated with disease outcomes in hospitalized patients with 2019 novel coronavirus disease. Chin Med J (Engl). 2020;133(9):1032-8. https://doi.org/10.1097/CM9.0000000000000775

24. Tetik BK, Tekinemre IG, Taș S. The effect of the COVID-19 pandemic on smoking cessation success. J Community Health. 2020;46(3):1-5. https://doi.org/10.1007/s10900-020-00880-2

25. Klemperer EM, West JC, Peasley-Miklus C, Villanti AC. Change in tobacco and electronic cigarette use and motivation to quit in response to COVID-19. Nicotine Tob Res. 2020;22(9):1662-3. https://doi.org/10.1093/ntr/ntaa072

26. Giulietti F, Filipponi A, Rosettani G, Giordano P, lacoacci C, Spannella F et al. Pharmacological approach to smoking cessation: an updated review for daily clinical practice. High Blood Press Cardiovasc Prev. 2020;27(5):349-62. https: / /doi.org/10.1007/s40292-020-00396-9

27. Malta DC, Szwarcwald CL, Barros MBA, Gomes CS, Machado ÍE, Souza Júnior PRB et al. A pandemia da COVID-19 e as mudanças no estilo de vida dos brasileiros adultos: um estudo transversal, 2020. Epidemiol Serv Saúde. 2020;29(4):1-13. https: / /doi.org/10.1590/S1679-49742020000400026

28. Santos MDV, Santos SV, Caccia-Bava MCGG. Prevalência de estratégias para cessação do uso do tabaco na atenção primária à saúde: uma revisão integrativa. Cienc Saúde Coletiva. 2019;24(2):563-72. https://doi.org/10.1590/1413-81232018242.27712016

29. Huber L, Cuadrado U, Fernandez-Megina R, Raw M, Marquizo AB, Romeo-Stuppy K. The impact of COVID-19 on the WHO FCTC, cessation, and tobacco policy. Tob Induc Dis. 2020;18:1-2. https://doi.org/10.18332/tid/130779

30. Ahluwalia IB, Myers M, Cohen JE. COVID19 pandemic: an opportunity for tobacco use cessation. Lancet Public Health. 2020;5(11):e577. https://doi.org/10.1016/S2468-2667(20)30236-X.

31. Ministério da Saúde (BR). Portaria conjunta № 10, de 16 de abril de 2020. Aprova o protocolo clínico e diretrizes terapêuticas do tabagismo. Diário Oficial União. 17 abr 2020.

\section{Contribuiçãa dos Autores}

Silva MVD, Monteiro MBA - Concepção, planejamento (desenho do estudo), aquisição, análise, interpretação dos dados e redação do trabalho. Sena AB - Aquisição, análise, interpretação dos dados e redação do trabalho. Martelli PJL, Heráclio IL, Ceballos AGC - Redação do trabalho. Todos os autores aprovaram a versão final do trabalho. 